\title{
Valoración de la mnemotecnia de la palabra clave para el aprendizaje de vocabulario en euskera: listas cortas de palabras
}

\author{
Valuating the keyword mnemonic in the learning of Basque language vocabulary: \\ short lists of words
}

\author{
Aitziber Goñi-Artola y María Ángeles González \\ Universidade da Coruña
}

\begin{abstract}
Resumen
Este trabajo tenía por objetivo analizar cómo los estudiantes de Educación Secundaria Obligatoria perciben y valoran la mnemotecnia de la palabra clave, en comparación con el método de repetición, cuando la utilizan para aprender listas cortas de palabras en euskera. Con ese fin, a un grupo de 102 alumnos de $1^{\circ}$ y $2^{\circ}$ curso de E. S.O. se le mostraron 14 palabras en euskera, con sus respectivas traducciones al castellano. La mitad de los estudiantes utilizaron el método de repetición para aprender la correspondencia entre las palabras, y la otra mitad,la mnemotecnia de la palabra clave. Los que aprendieron las palabras utilizando la mnemotecnia de la palabra clave ofrecieron puntuaciones significativamente superiores en percepción de facilidad de la estrategia y en agrado hacia la tarea.

Palabras clave: Mnemotecnia, método de la palabra clave, aprendizaje de vocabulario.
\end{abstract}

\section{Abstract}

This work aimed to analyze how students of Secondary Education perceive and value the keyword mnemonic, compared with the rote method, when used it to learn short lists of words in Basque. To that end, they were shown 14 words in Basque to a group of 102 students of 1st and 2nd year of ESO, with their translations into Spanish .Half of the students used the method of repetition to learn the correspondence between words, and the other half, the keyword mnemonic. Those who learned the words using the keyword mnemonic offered significantly higher scores in perceived ease of the strategy and pleasure to the task.

Keywords: Mnemonics, Keyword mnemonic, vocabulary learning.

\section{Introducción}

La mnemotecnia de la palabra clave es una regla mnemotécnica que principalmente se usa para el aprendizaje de vocabulario de segundos idiomas (Ashoori, 2012; Atkinson y Raugh, 1975; Baleghizade y Ashoori, 2010; Campos, Rodriguez-Pinal y Pérez-Fabello, 2013; Fritz, Morris, Action, Voelkel y Etkind, 2007). La regla mnemotecnia consta de dos fases: una primera donde se relaciona la palabra que se quiere aprender con una palaba conocida de sonido parecido (palabra clave), y una segunda fase donde se forma una imagen mental interactiva relacionando la palabra clave con la traducción de la palabra que debe ser aprendida.

Su eficacia se ha demostrado en muchos idiomas. Inicialmente fue utilizada con términos en ruso (Atkinson y Raugh, 1975) y en español (Raugh y Atkinson, 1975). Los sujetos eran estudiantes hablantes nativos de inglés que no habían estudiado nunca ruso ni español, respectivamente. Desde entonces se ha intentado demostrar la eficacia de la mnemotecnia de la palabra clave en diferentes idiomas. Se ha visto que es efectiva con el inglés (Ashoori, 2012; Tabatabaei y Hejazi, 2011), el español (Van Helly Mahn, 1997; Wyra, Lawson y Hungi, 2007), el latín (Campos y Amor, 2004; Campos, Amor y González, 2002; Campos, Pérez-Fabello y Camino, 2010), o el alemán (Fritz et al., 2007), entre otros idiomas. También se ha usado con el inglés, limitándose a vocabulario de negocios y economía por parte de hablantes del idioma árabe (Abdel-Majeed, 2000).

Fritz et al. (2007) realizaron un trabajo donde se comparaba la eficacia de la mnemotecnia de la palabra clave, de la práctica de recuperación, y del aprendizaje por repetición, en idiomas como el turco, japonés, hebreo, galés, italiano, ruso y polaco. Los participantes tenían que aprender 15 traducciones, que se presentaron a través de cintas de audio. El recuerdo se produjo tanto inmediatamente como tres días después. No hubo diferencias significativas entre el grupo que utilizó la mnemotecnia de la palabra clave y la práctica de recuperación,siendo el recuerdo en ambos grupos sustancialmente superior a los que aprendían por repetición.

La mnemotecnia de la palabra clave se ha utilizado fundamentalmente para el aprendizaje de vocabulario de una lengua extranjera o no conocida, pero también se ha comprobado su eficacia en palabras del mismo idioma con baja frecuencia de uso (Campos et al., 2013).Además, se ha probado la eficacia de la estrategia con otro tipo de material, como términos científicos y médicos (Brarhler y Walker, 2008), vocabulario matemático (Brown, 2007), nombres de fobias (Carney y Levin, 2008) o, como se ha comentado antes, vocabulario de negocios y economía (Abed-Majeed, 2000). 
También se ha investigado si la mnemotecnia de la palabra clave es eficaz en diferentes grupos de edad, ya que la mayoría de las investigaciones relacionadas con la mnemotecnia, y específicamente con la mnemotecnia de la palabra clave, se han realizado con sujetos en edad escolar. Campos, Camino y Pérez-Fabello (2010) utilizaron una muestra con una media de edad de 58.9 años para ver la eficacia de la mnemotecnia de la palabra clave frente al método de repetición. Encontraron que la mnemotecnia de la palabra clave era más eficaz que la repetición en listas cortas y en listas largas, con palabras altas y bajas en imagen.

Siguiendo con diferentes tipos de muestras, también se ha encontrado que la mnemotecnia de la palabra clave es más eficaz que otras técnicasen personas con dificultades de aprendizaje (Jitendra, Edwards, Sacks, y Jacobson, 2004).

Una limitación de la mnemotecnia de la palabra clave es la adecuación de la palabra clave utilizada. En la mayoría de los estudios sobre este método los investigadores facilitan la palabra clave y una sugerencia de imagen interactiva. Sin embargo, si alguien desea utilizar la mnemotecnia más allá de ese conjunto de palabras, debe crear sus propias conversiones entre los idiomas. Esto puede ser interesante y tal vez beneficioso, en sí mismo, para el aprendizaje de las traducciones, pero también exige esfuerzo, creatividad y tiempo. Además, es probable que algunas palabras no se presten a este enfoque (Fritz et al. 2007).

El debate sobre la manera de generar la palabra clave todavía sigue vigente. Existe controversia sobre si la palabra clave la debe generar el sujeto o es el experimentador el que debe facilitarla. Campos y Amor (2004), Campos et al. (2002) y Campos, González y Amor (2004) crearon un nuevo método de generar la palabra clave donde la palabra clave la genera un grupo de iguales que no participa en el experimento. El grupo de iguales lo compondrían sujetos de características socioculturales y edad similares a los sujetos experimentales. Se encontró que los resultados mejoraban, tanto a corto como a largo plazo, cuando la palabra clave la generaban los compañeros.

Como se ha visto, el método de la mnemotecnia de la palabra clave es una estrategia ampliamente investigada, y su eficacia comprobada en diferentes idiomas, grupos de edad y personas, y con diferente tipo de material. Pero los beneficios de esta estrategia no solo se limitan a la mejora del rendimiento. La aplicación de la mnemotecnia de la palabra clave, además de mejorar el rendimiento de los sujetos, conlleva también otro tipo de beneficios, tanto para el discente, como para el docente. La monotonía, muchas veces causa del escaso interés y constancia en el estudio, queda anulada con la ejecución de la técnica. El aprendizaje realizado bajo estas directrices resulta mucho más entretenido, divertido y motivador para el sujeto (González, Amor y Campos, 2003).

Hauptmann (2004) se planteó si la mnemotecnia de la palabra clave contribuía al disfrute o no del aprendizaje, y encontró que tenía un efecto beneficioso sobre la motivación de los alumnos. Encontró que los beneficios de esta mnemotecnia no solo se limitaban al recuerdo, sino que tenía un efecto beneficioso sobre la motivación de los alumnos.

Las investigaciones que estudian la eficacia en el recuerdo de la mnemotecnia de la palabra clave son abundantes, pero no son tantas las que la relacionan con variables como la motivación, la diversión o el entretenimiento. Por ello, en este estudio se plantea como objetivoanalizar como los alumnos de E.S.O. perciben la facilidad y el agrado hacia la mnemotecnia de la palabra clave en comparación con el método de repetición, cuando la utilizan para aprender listas cortas de palabras (14 palabras) en euskera.

\section{Método}

\section{Participantes}

En el estudio participó un grupo de 102 estudiantes de $1^{\circ}$ y $2^{\circ}$ curso de Educación Secundaria Obligatoria de varios institutos de A Coruña (57 hombres y 45 mujeres), con una media de edad de 13.09 años (SD = .95).

\section{Material}

Utilizamos una lista de 14 palabras en euskera (7 altas en imagen y 7 bajas en imagen) y su correspondiente traducción al castellano. También 14 palabras clave para cada una de las palabras en euskera, y 14 frases interactivas relacionando la palabra clave con la correspondiente traducción al castellano de la palabra en euskera.

Elaboramos un cuestionario de valoración del método de aprendizaje, que fue utilizado en esta investigación para obtener los datos de los participantes en las variables estudiadas. En concreto, en este trabajo se utilizaron los ítems referidos a la dificultad-facilidad percibida, y al agrado hacia la tarea de aprendizaje utilizando la estrategia correspondiente. Estos ítems se valoraron en una escala tipo Likertde 7 niveles (siendo el 1: muy difícil o mínimo agrado, dependiendo del ítem en cuestión, y el 7: muy fácil o máximo agrado, respectivamente).

\section{Procedimiento}

Las palabras del estudio se seleccionaron a partir de las publicadas por Valle (1998). En un primer paso, dentro del apartado Normas de imaginabilidad (Orden decreciente), se fueron anotando 1 de cada 10 palabras en el orden que aparecen, siempre que el valor de imagen fuera mayor que 5.20 (consideradas altas en imagen) o menor de 4.21 (consideradas bajas en imagen). Se tradujeron esas palabras al euskera y se rechazaron todas las palabras que sonasen o escribiesen de forma similar en ambos idiomas. De los sustantivos que conformaban ese conjunto de palabras, se seleccionaron al azar 16 palabras altas en imagen y 16 palabras bajas en imagen, 32 sustantivos en total.

Se presentaron las 32 palabras en euskera, y sus traducciones, a un grupo de sujetos de características similares a los participantes, en este caso 48 alumnos de $1^{\circ}$ y $2^{\circ}$ de E.S.O. Dichos sujetos elaboraron, para cada palabra en euskera, una palabra clave y una frase que describiera una imagen mental en la que interactuasen la 
palabra clave con el significado de la palabra en euskera. De todas las palabras clave generadas por este grupo de compañeros, se eligieron las más frecuentes (las de mayor concordancia entre los sujetos). Para este estudio, finalmente se contó con 14 palabras en euskera(7 altas en imagen y 7 bajas en imagen). Una vez seleccionadastambién las palabras clave para estos 14 términos en euskera, se escogieron las frases interactivas que aparecían con mayor frecuencia.

El grupo de 102 participantesque participaban en el presente estudio fue dividido al azar en dos grupos, grupo de repetición (52 participantes) y grupo de mnemotecnia de la palabra clave (50 participantes). A todos ellos, en sus respectivas clases, se les presentóla lista final de 14 palabras en euskera, en diapositivas a través de un proyector, para que aprendieran su traducción al castellano. Para los que aprendían a través del método de repetición, cada diapositiva estaba compuesta por una palabra en euskera y su correspondiente traducción al castellano. Para los que aprendían a través de la mnemotecnia de la palabra clave, cada diapositiva constaba de la palabra en euskera, su correspondiente traducción al español, la palabra clave y la frase interactiva correspondiente. Para ambos grupos, cada diapositiva permanecía 15 segundos en pantalla y cambiaba a la siguiente automáticamente.

Una vez finalizada la sesión de aprendizaje de las palabras, se les aplicó el cuestionario en el que se les preguntaba acerca de la dificultad-facilidad percibida en la tarea con el uso de esa estrategia, y sobre su agrado hacia la misma.

\section{Resultados}

Con el fin de averiguar si los estudiantes que aprendieron la lista corta de palabras en euskera utilizando la mnemotecnia de la palabra clave, percibían y valoraban este método de forma diferente de los que la aprendieron con el método de repetición, se efectuó un Análisis Multivariante de Varianza (MANOVA), teniendo como variable independiente la estrategia de aprendizaje empleado, y como variables dependientes, las puntuaciones de los participantes en percepción de la facilidad de la estrategia de aprendizaje empleada, y en agrado hacia la tarea al utilizar esa estrategia. Las medias y desviaciones típicas en estas dos variables para ambas estrategias de aprendizaje aparecen en la tabla 1.

Tabla 1.

Medias y desviaciones típicas en percepción de Facilidad y Agrado en función del método empleado para aprender

\begin{tabular}{lcccccc}
\hline & \multicolumn{2}{c}{$\begin{array}{c}\text { Palabra } \\
\text { clave }\end{array}$} & \multicolumn{2}{c}{ Repetición } & \multicolumn{2}{c}{ Total } \\
\cline { 2 - 7 } & $M$ & $S D$ & $M$ & $S D$ & $M$ & $S D$ \\
\hline Facilidad & 4.18 & 1.24 & 3.42 & 1.27 & 3.79 & 1.31 \\
Agrado & 4.86 & 1.64 & 3.85 & 1.86 & 4.34 & 1.82 \\
\hline
\end{tabular}

Encontramos una Lambda de Wilks $=.89, \mathrm{~F}(2,99)=$ 5.940, $\mathrm{p}<.01$, que indicó que existía influencia del método de aprendizaje en la valoración que los estudiantes efectuaron (percepción de facilidad y en el agrado hacia la tarea de aprendizaje). Los posteriores Análisis Univariados mostraron que existe una diferencia significativa en la percepción de facilidad $F(1,100)=$ 9.239, $p<.01$, entre los que aprendieron las palabras con la mnemotecnia de la palabra clave $(M=4.18 ; S D=1.24)$ y los que las aprendieron a través del método de repetición $(M=3.42 ; S D=1.27)$. También el método utilizado por los participantes influyó en el agrado hacia la tarea de aprendizaje, $F(1,100)=8.485, p<.01$. Los participantes que aprendieron las palabras mediante la mnemotecnia de la palabra clave manifestaron un mayor agrado hacia la tarea $(M=4.86 ; S D=1.64)$ que los estudiantes que las aprendieron a través del método de repetición $(M=3.85 ; S D=1.86)$.

\section{Discusión}

Los resultados obtenidos muestran que los alumnos que aprendieron el significado de una lista corta de palabras en euskera a través de la mnemotecnia de la palabra clave, perciben la estrategia de aprendizaje en esta tarea como más fácil y más agradable que los alumnos que aprenden las mismas palabras a través del método de repetición. El hecho de que el material a aprender fueran palabras en euskera, un idioma sobre el que no nos constan datos en relación con esta estrategia, suma interés a este resultado.

La mnemotecnia de la palabra clave, además de ser un método eficaz para el aprendizaje de segundos idiomas y vocabulario técnico, o de baja frecuencia en nuestro propio idioma, se muestra así como una estrategia que tiene una respuesta positiva por parte del estudiante. Este resultado es relevante, dado que la motivación es quizás la preocupación más importante para los profesores de idiomas, que intentan incluir actividades de motivación en su clase, incluso cuando su valor real de aprendizaje es mínimo (Hauptmann, 2004).

Sin duda, además de la información sobre la valoración de la mnemotecnia de la palabra clave en el aprendizaje de palabras en euskera, resultará de utilidad contar con datos sobre la eficacia de la estrategia en el aprendizaje de vocabulario en este idioma. Pero, dado quelas investigaciones sobre otras ventajas, al margen de la mejora del recuerdo, son escasas, creemos que éste es un campo en el que se debería seguir investigando, pues factores como la motivación, interés, confianza facilidad o agrado están relacionados con un mejor y más satisfactorio aprendizaje(González et al., 2003).

\section{Referencias}

Abdel-Majeed, M. M. (2000). The keyword method: a powerful memory aid to vocabulary learning in the EFL classroom (An Experimental Study). The E.R.C. Journal, 17, 9-27.

Ashoori, A. (2012). Recall of foreign-language vocabulary: effects of keyword, context and word list instructional strategies on long-term vocabulary recall 
of EFL learners. Journal of Theory and Practice in Education, 8, 54-71.

Atkinson, R.C. y Raugh, M.R. (1975). An application of the mnemonic keyword method to the acquisition of a Russian vocabulary. Journal of Experimental Psychology: Human Learning and Memory, 104, 126-133.

Baleghizade, S. y Ashoori, A. (2010). The effect of keyword on word list method on immediate vocabulary retention of EFL learners. Pakistan Journal of Social Sciences, 30 (2), 251-261.

Brahler, J. C. y Walker, D. (2008). Learning scientific and medical terminology with a mnemonic strategy using an illogical association technique. Advances in Physiology Education, 32(3), 219-224.

Brown, G. D. (2007). Mathematics vocabulary instruction for current non-proficient students with and without IEPs: A study of three methods of instruction. Dissertation Abstracts International, 69(1-A), 1 76-1 76.

Campos, A. y Amor, A. (2004). Among-subject concordance in keyword generation and its effect on recall: A preliminary study. Imagination, Cognition and Personality, 24(4), 347-354.

Campos, A., Amor, A. y González, M. A. (2002). Presentation of keywords by means of interactive drawings. The Spanish Journal of Psychology, 5 (2), 102-109.

Campos, A., Camino, E. y Pérez-Fabello, M. J. (2010). Aprendizaje de vocabulario con alta y baja viveza de imagen mediante la mnemotecnia de la palabra clave. Revista Galego-Portuguesa de Psicoloxía e Educación, 18(2), 67-77.

Campos, A., González, M. A., y Amor, A. (2004). Different strategies for keywords generation. Journal of Mental Imagery, 28(3\&4), 51-58.

Campos, A., Pérez-Fabello, M. J. \& Camino, E. (2010). Eficacia de la mnemotecnia de la palabra clave en personas adultas. Psicothema, 22 (4), 752-757.

Campos, A., Rodríguez-Pinal, M. D. y Pérez-Fabello, M. J. (2013). Aprendizaje del idioma gallego mediante la mnemotecnia de la palabra clave, en personas bilingües, altas y bajas en imagen. Revista de Investigación en Educación, 11(2), 50-59.
Carney, R. N., y Levin, J. R. (2008). Conquering mnemonophobia, with help from three practical measures of memory and application. Teaching of Psychology, 35(3), 176-183.

Fritz, C. O., Morris, P. E., Acton, M., Voelkel, A. R. y Etkind, R. (2007). Comparing and combining retrieval practice and the keyword mnemonic for foreign vocabulary learning. Applied Cognitive Psychology, 21, 499-526. doi:10.1002/acp.1287

González, M. A., Amor, A. y Campos, A. (2003). La mnemotecnia de la palabra clave. A Coruña: Servicio de Publicaciones de la Universidad de A Coruña.

Hauptman, J. (2004). The effect of the integrated keyword method on vocabulary retention and motivation. Ph.D. Thesis. University of Leicester, Leicester, Reino Unido.

Jitendra, A. K., Edwards, L. L., Sacks, G. y Jacobson, L. A. (2004). What research says about vocabulary instruction for students with learning disabilities. Exceptional Children, 70(3), 299.

Raugh, M. R. y Atkinson, R. C. (1975). A mnemonic method for the learning of a second language vocabulary. Journal of Educational Psychology, 67, 1-16.

Tabatabaei, O. y Hejazi, N. H. (2011) Gender differences in vocabulary instruction using keyword method (Linguistic Mnemonics). Canadian Social Science, 7(5), 198-204. doi:10.3968/J.css.1923669720110705.465

Valle, F. (1998). Normas de imaginabilidad. Oviedo: Servicio de Publicaciones de la Universidad de Oviedo.

VanHell, J. G. y Mahn, A. C. (1997). Keyword mnemonic versus rote rehearsal: learning concrete and abstract foreign word by experienced and inexperienced learners. Language Learning, 47 (3), 507-546.

Wyra, M., Lawson, M. J. y Hungi, N. (2007). The mnemonic keyword method: The effects of bidirectional retrieval training and of ability to image on foreign languag evocabulary recall. Learning and Instruction, 17, 360-371. 